\section{SNR enhancement of Raman-based long-range distributed temperature sensors using cyclic Simplex codes}

F. Baronti, A. Lazzeri, R. Roncella, R. Saletti, A. Signorini, M.A. Soto, G. Bolognini and F. Di Pasquale

Presented is a novel approach for enhancing the signal-to-noise ratio (SNR) of optical fibre distributed temperature sensors. The proposed method is based on cyclic codes and it can be implemented by highpower lasers, which are required for long-range applications but are unsuitable for conventional coding techniques. The expected performance improvement, in terms of temperature resolution, sensing range and measurement time, is confirmed by simulations.

Introduction: Optical fibre distributed temperature sensors based on spontaneous Raman scattering (RDTS) have potential application in several industrial and scientific fields for reliable temperature monitoring over long distances (up to a few tens of kilometres) [1]. They are based on optical time-domain reflectometry (OTDR): an optical pulse is sent along a sensing fibre and the temperature-dependent backscattered Stokes (S) and anti-Stokes (AS) photons are acquired as a function of time. The collected samples are then processed to extract the temperature profile.

The main issue to face in long-range RDTS design is the low signalto-noise ratio (SNR) due to the weakness of the backscattered S and AS traces, which generally exhibit optical power levels lower than a few $\mathrm{nW}$. The energy of the launched pulse is indeed bounded by the targeted spatial resolution, which implies a small pulse width, and by the threshold for the onset of the fibre nonlinearities, which upper bounds the pulse peak level. To reduce the noise power introduced by the optical/electrical receiver, and reach a temperature resolution of a few degrees at the fibre-end, trace averaging is typically employed. However, the greater the number of averages, the longer the measurement time, which might become unsuitable for several applications requiring short response times.

Coding techniques based on Simplex or Golay codes exploit a set of different sequences (i.e. codes) of short (about $10 \mathrm{~ns}$ ) NRZ laser pulses to increase the launched energy without impairing the spatial resolution [2]. However, the required high repetition rate of the laser pulses - a hundred of $\mathrm{MHz}$ for a meter-scale spatial resolution - is not achievable by high peak power lasers, such as rare-earth doped fibre or passive Q-switched ones, which feature a maximum repetition rate of some hundreds of $\mathrm{kHz}$ [3].

Here, a new coding technique tailored to high-power pulsed lasers is proposed [4]. The basic idea is to periodically sense the probing fibre with a multi-pulse pattern the repetition period of which is equal to the fibre round-trip time. This way, the pattern results as a code spread along the whole fibre, with a bit time inversely proportional to the code length. The pulse width can be kept in the order of $10 \mathrm{~ns}$ to guarantee a meter-scale spatial resolution and the peak power can be set close to the nonlinear effect threshold.

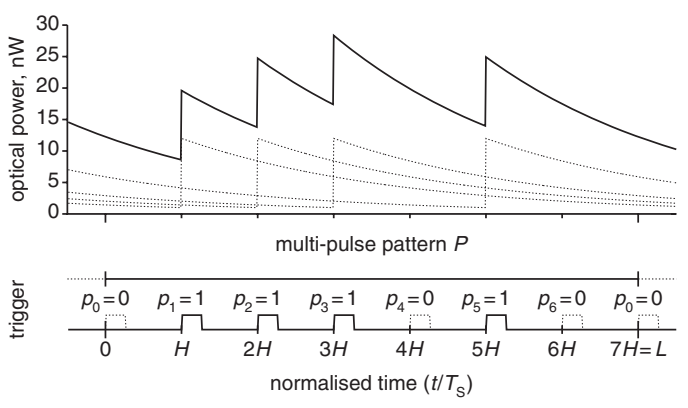

Fig. 1 Trace overlap in case of multi-pulse technique with $M=7$ and $P=$ $\{0,1,1,1,0,1,0\}$

Solid trace is sum of dashed ones, which represent fibre responses to laser pulses launched according to pattern repetition

Multi-pulse technique: First, let us consider an $M$-bit binary pattern $P=$ $\left\{p_{0}, \ldots, p_{M-1}\right\}$, with $p_{j}=0,1$ for $j=0, \ldots, M-1$. Suppose that the acquisition of the backscattered trace is divided into $M$ consecutive intervals, correspondingly to the $M$-bit pattern, and that a laser pulse is sent to the probing fibre at the beginning of each interval if the relevant pattern bit is 1 . Suppose also that this triggering scheme is being repeated periodically. According to $P$, some delayed backscattered traces overlap along the fibre, as shown in Fig. 1 for $M=7$ and $P=\{0,1$, $1,1,0,1,0\}$.

Given the system sampling period $T_{\mathrm{S}}$, the length of the backscattered trace can be expressed as the number $L$ of its samples. Now let $H$ be the number of samples within each of the $M$ intervals, so that $L=H \times M$. By defining a further index $i=0, \ldots,(H-1)$, we indicate with $y[i+j H]$ the array of the acquired samples, and with $x[i+j H]$ the array of the single-pulse response samples to be recovered. This way, $j$ scans intervals, whereas $i$ scans the samples within the $j$ th interval. It can be observed from Fig. 1 that each $y[i+j H]$ is due to the contribution of the backscattered traces originated by the pulses launched in the $j$ th interval and in the $M-1$ previous ones. Hence, the following relationship holds for $y$ and $x$ samples

$$
y[i+j H]=\sum_{k=0}^{M-1} p_{\left[j-\left.k\right|_{M}\right.} \times[i+k H]
$$

In particular, for a given $i,(1)$ turns into a linear system of $M$ equations (one for each $j$ value), the coefficient matrix of which is cyclic. In fact, its first row $(j=0)$ is $\left\{p_{0}, p_{M-1}, \ldots, p_{2}, p_{1}\right\}$ and any other row is the right-shifted copy of the previous one.

From a noise point of view, $y$ and $x$ samples can be safely considered as uncorrelated random variables. The noise affecting the recovered $x$ samples is determined by the linear system itself, i.e. by the inverse of the system coefficient matrix. Hence, $P$ should be chosen so that a good noise reduction can be achieved. To this end, as the coefficient matrix is cyclic by construction, it is sufficient to use a matrix derived from the cyclic code theory and choose the pattern $P$ accordingly.

Considering the cyclic Simplex codes, a cyclic binary coefficient matrix of order $M=4 n-1$ with $n=1,2, \ldots$ can be built using the method reported in [5]. Being $\sigma_{Y}$ and $\sigma_{X}$ the standard deviation of any $y$ and $x$ sample, respectively, the achieved SNR enhancement, i.e. the coding gain $C_{\text {gain }}$, is given by the following well-known relation [2]

$$
C_{\text {gain }}=\frac{\sigma_{Y}}{\sigma_{X}}=\frac{M+1}{2 \sqrt{M}}
$$

Finally, we point out that the implementation of such a coding technique requires only a few changes in the architecture of a typical RDTS, as highlighted in Fig. 2. As the trigger generation and the trace decoding blocks are usually implemented on programmable hardware (e.g. on an FPGA), firmware, or high-level software, they can be easily upgraded. Moreover, the multi-pulse pattern can be obtained by triggering the laser at a fixed rate and by implementing the cyclic code through an external modulator (e.g. an acousto-optic one), which is in charge of filtering out a pulse if the corresponding bit code is equal to 0 . This way, the pulsed laser operates at a constant frequency, which guarantees good repeatability of the generated pulse shape and peak power [3].

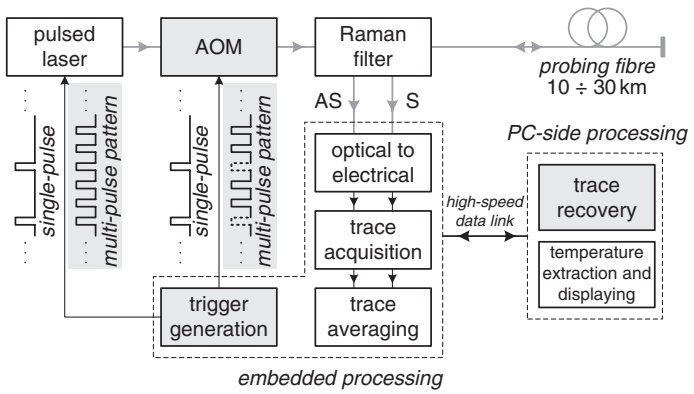

Fig. 2 Typical RDTS architecture

Shadowed blocks refer to multi-pulse technique implementation

Application example: A mathematical model derived from a real RDTS [6] (refer again to Fig. 2 for its scheme) has been used to simulate and compare the performance of both multi-pulse and conventional singlepulse techniques. The largest feasible code length is $M_{\max }=T_{\mathrm{R}} \times$ $f_{\max }$, where $T_{\mathrm{R}}$ is the fibre round-trip time and $f_{\max }$ is the maximum pulse repetition rate of the laser.

The simulations have been performed considering a $30 \mathrm{~km}$ fibre length $\left(T_{\mathrm{R}} \simeq 300 \mu \mathrm{s}\right)$ and $f_{\max }=300 \mathrm{kHz}$. Using cyclic Simplex 
codes, we chose the largest $M \leq M_{\max }$ known by the code theory, i.e. $M=83(n=21)$. The effects of white Gaussian noise were included according to formerly collected experimental data [6]. The sensing fibre is maintained at the room temperature of $300 \mathrm{~K}$, apart from a short track between 14.5 and $15 \mathrm{~km}$ where the temperature is equal to $323 \mathrm{~K}$. Figs. $3 a$ and $b$ show the simulation results for the single-pulse and the multi-pulse cases, respectively. The temperature resolution, i.e. the standard deviation $\sigma_{T}$ of the reconstructed temperatures at a given distance, has been evaluated by simulating 1000 noisy $\mathrm{S}$ and AS traces. Fig. $3 c$ clearly shows the improvement provided by the multi-pulse technique.
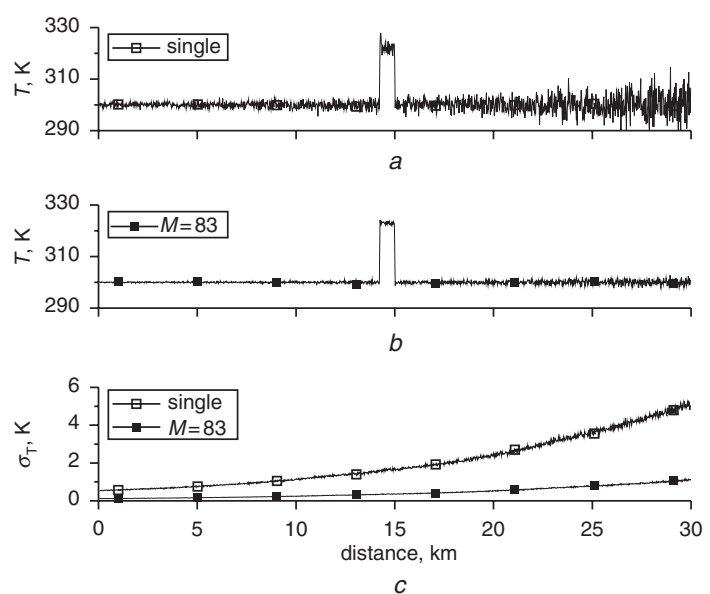

Fig. 3 Examples of reconstructed temperature profiles after $100 k$ averages for single-pulse $(M=1)$ (Fig. 3a) and multi-pulse $(M=83)$ (Fig. 3b) RDTS, and relevant temperature resolutions (Fig. 3c)

Finally, Fig. 4 shows $\sigma_{T}$ at the fibre-end against $M$ for some measurement times, i.e. for a different number of averages. It is worth noticing that a $1.1 \mathrm{~K}$ temperature resolution is achieved by a conventional RDTS (i.e. with $M=1)$ after $610.3 \mathrm{~s}(2.125 \mathrm{M}$ avgs), time that can be shortened to $28.7 \mathrm{~s}$ ( $100 \mathrm{k}$ avgs) by applying the proposed cyclic coding technique with $M=83$. The time reduction factor is 21.26 . This implies an SNR enhancement of about $\sqrt{21.26}=4.61$, which is in good agreement with the theoretical value of $\left.C_{\text {gain }}\right|_{M=83}=4.58$. Referring to $100 \mathrm{k}$ avgs, the achievable $\sigma_{T}$ for the conventional RDTS is $5.1 \mathrm{~K}$, a value that can be reached in just $1.4 \mathrm{~s}(4.8 \mathrm{k}$ avgs $)$ with the considered coding method.

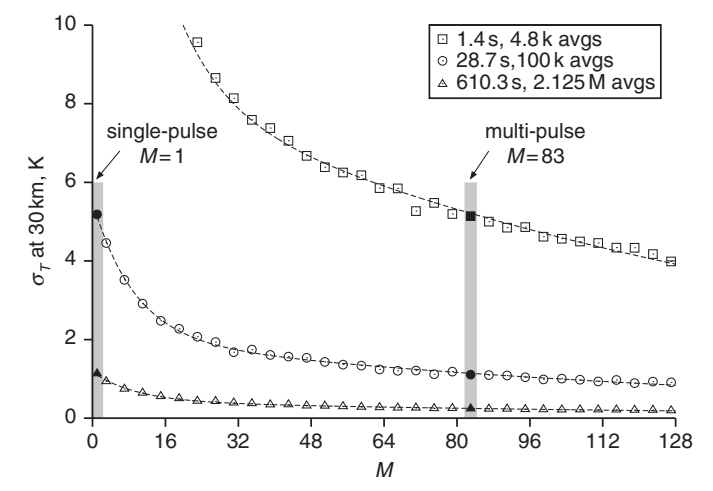

Fig. 4 Temperature resolution at fibre-end against code length, for different measurement times
Conclusion: A new multi-pulse pattern technique based on cyclic codes, suitable for high-power lasers, is presented. The performance improvement of its application to long-range RDTS systems has been verified by numerical simulations and compared to conventional RDTS performance. Simulation results show significant advantages in terms of temperature resolution improvement, measurement time reduction, and sensing range extension, with respect to conventional RDTS systems.

(C) The Institution of Engineering and Technology 2010

31 May 2010

doi: 10.1049/el.2010.1484

F. Baronti, A. Lazzeri, R. Roncella and R. Saletti (Department of Information Engineering, University of Pisa, via G. Caruso 16, Pisa 56126, Italy)

E-mail: f.baronti@iet.unipi.it

A. Signorini, M.A. Soto, G. Bolognini and F. Di Pasquale (Scuola Superiore Sant'Anna, via G. Moruzzi 1, Pisa 56124, Italy)

\section{References}

1 Adachi, S.: 'Distributed optical fiber sensors and their applications'. Proc. of SICE Annual Conf., Tokyo, Japan, 2008, pp. 329-333

2 Jones, M.D.: 'Using Simplex codes to improve OTDR sensitivity', IEEE Photonics Technol. Lett., 1993, 5, (7), pp. 822-824

3 Myslinski, P., Chrostowski, J., Koningstein, J., and Simpson, J.: 'High power Q-switched erbium doped fiber laser', IEEE J. Quantum Electron., 1992, 28, (1), pp. 371-377

4 Bolognini, G., Di Pasquale, F., Baronti, F., Lazzeri, A., and Roncella, R.: Italian Patent Pending PI2010A000 046, 9 April 2010

5 Song, H., and Golomb, S.: 'Some new constructions for Simplex codes', IEEE Trans. Inf. Theory, 1994, 40, (2), pp. 504-507

6 Soto, M.A., Sahu, P.K., Faralli, S., Bolognini, G., Di Pasquale, F., Nebendahl, B., and Rueck, C.: 'Distributed temperature sensor system based on Raman scattering using correlation-codes', Electron. Lett., 2007, 43, (16), pp. 862-864 